Michael Lösler* und Cornelia Eschelbach

\title{
Orthogonale Regression - Realität oder Isotropie?
}

\section{Orthogonal regression - reality or isotropy?}

https://doi.org/10.1515/teme-2020-0063

Eingang 29. Juli 2020; angenommen 18. August 2020

Zusammenfassung: Nicht nur in der Metrologie wird für die Analyse von Daten auf eine orthogonale Regression zurückgegriffen. Bei der orthogonalen Regression stehen die zu minimierenden Verbesserungen senkrecht auf der Funktion, weshalb dem Verfahren häufig ein hoher Anschauungsgrad beigemessen wird. In diesem Beitrag werden einige Eigenschaften der orthogonalen Regression untersucht und geprüft, ob diese exklusiv diesem Verfahren zuzuordnen sind oder ob es sich lediglich um übertragene Eigenschaften eines allgemeinen Optimierungsproblems handelt. Ergänzend zu den Ausführungen von Kolaczia [9] soll die Frage nach der Eignung dieses Verfahrens für die Messtechnik beantwortet werden.

Schlagwörter: Orthogonal-Distance-Fitting, Orthogonale Regression, Sequentielle quadratische Programmierung, Regressionsanalyse, Messtechnik.

Abstract: The orthogonal distance fitting is a common approach not only in metrology. Minimizing the orthogonal distances is often called as the most natural error criterion in least squares adjustments because such a derived solution provides a clear physical meaning. In this contribution, some of the implied properties of the orthogonal regression are investigated. It is evaluated whether these properties hold for a general optimization problem or whether these are exclusive properties of the orthogonal regression. Following Kolaczia [9], the goal of our investigation is to answer the question, whenever the orthogonal distance fitting should be applied in metrology.

Keywords: Orthogonal-distance-fitting, orthogonal regression, sequential quadratic programming, regression analysis, metrology.

Für Maria Hennes

*Korrespondenzautor: Michael Lösler, Frankfurt University of Applied Sciences, Faculty of Architecture, Civil Engineering and Geomatics, Laboratory for Industrial Metrology, Nibelungenplatz 1, D-60318 Frankfurt am Main, Germany, E-Mail:

michael.loesler@fb1.fra-uas.de, ORCID:

https://orcid.org/0000-0002-1979-263X

Cornelia Eschelbach, Frankfurt University of Applied Sciences, Faculty of Architecture, Civil Engineering and Geomatics, Laboratory for Industrial Metrology, Nibelungenplatz 1, D-60318 Frankfurt am Main, Germany, ORCID: https://orcid.org/0000-0003-4959-8712

\section{Einleitung}

Für die sachgerechte Analyse von erhobenen Daten wird in den messenden Disziplinen häufig auf eine Ausgleichungsrechnung nach der Methode der kleinsten Quadratsumme zurückgegriffen. Neben den eigentlichen Zielgrößen, den Modellparametern, liefert die Ausgleichungsrechnung weitere Kenngrößen und Zuverlässigkeitsmaße, die eine statistische Bewertung der Schätzung ermöglichen. Die Ausgleichungsrechnung zählt somit zu den wichtigsten Analysewerkzeugen um bspw. Veränderungen an einem Messobjekt in der Kongruenzanalyse zu detektieren, um die Formtreue eines Bauteils zu validieren oder um die Position und Ausrichtung einzelner Elemente im Maschinen- und Anlagenbau zu justieren.

Im Reverse Engineering werden häufig Kurven bzw. Flächen zweiter Ordnung (Quadrik) wie bspw. Geraden, Kugeln, Ebenen oder Zylinder verwendet. Die Bestimmung der Formparameter erfolgt hierbei mitunter durch Minimierung der orthogonalen Abstände, d.h., den Normalenabständen zwischen der Quadrik und den gemessenen Punkten. In der Literatur ist diese Schätzung auch als Orthogonal-Distance-Fitting bzw. orthogonale Regression bekannt. Sie wurde erstmals 1840 von Weisbach [18] beschrieben, der zur Bestimmung der Hauptrichtung von Lagerstädten im Markscheidewesen auf eine Regressionsgerade zurückgriff, die sowohl zufällige Messabweichungen in der $x$ - als auch in der $y$-Komponente der Punkte berücksichtigt.

Die Verbreitung der orthogonalen Regression liegt vornehmlich in ihrem hohen Anschauungsgrad [15]. Wijewickrema et al. [19] bezeichnen die Minimierung der Normalenabstände als ein natürliches Bewertungsmaß, da den geschätzten Modellparametern eine physische oder geometrische Bedeutung zugeordnet werden kann [6]. Insbesondere sind die intrinsischen Formparameter wie bspw. der Radius einer Kugel invariant bzgl. einer Koordinatentransformation [1, S. 11]. Weiterhin existieren für einige Geometrien direkte Lösungsverfahren, die den Einsatz der orthogonalen Regression in der Praxis motivieren [10, 14].

Andererseits hinterfragt Kolaczia [9] die Eignung der orthogonalen Regression für die sachgerechte Datenanalyse in der industriellen Messtechnik. Zum einen werden durch die Minimierung der orthogonalen Abstände die einzelnen Koordinatenkomponenten unterschiedlich 
stark berücksichtigt. Zum anderen sind die Ergebnisse nicht skalierungsinvariant, wenn bspw. zwischen Maßeinheiten umgerechnet wird. Kolaczia [9] resümiert, dass die Anwendung der orthogonalen Regression in der Metrologie ungeeignet ist, da die Ergebnisse von der Wahl der Maßeinheiten zwingend unabhängig sein müssen.

In der Koordinatenmesstechnik wird eine Reihe von Messinstrumenten eingesetzt, die keine kartesischen Koordinaten registrieren [5]. Der Lasertracker ist bspw. ein polares Messinstrument, welches eine Strecke und zwei Winkel registriert. Koch \& Kargoll [8] zeigen, dass die Ergebnisse einer Parametereschätzung unabhängig von der gewählten Koordinatendarstellung sind. Im Gegensatz dazu weisen Holst et al. [6] darauf hin, dass es für eine Minimierung der orthogonalen Abstände kartesische Koordinaten bedarf. Die Autoren schlagen vor, die originär erfassten Polarkoordinaten in korrespondierende kartesische Koordinaten umzurechnen und die orthogonale Regression auf der Basis der kartesischen Koordinaten durchzuführen.

In diesem Beitrag wird die Gültigkeit dieser z. T. konträren Eigenschaften und Voraussetzungen der orthogonalen Regression näher untersucht. In Anlehnung an die Untersuchungen von Kolaczia [9] wird geprüft, ob die Anwendung der orthogonalen Regression in der Metrologie empfehlenswert ist. Um weitgehend unabhängig von einem speziellen Optimierungsproblem zu sein, erfolgt die Schätzung der Modellparameter in diesem Beitrag mittels sequentieller quadratischer Programmierung (SQP). Die SQP zählt zu den Standardverfahren in der numerischen Optimierung und wird insbesondere für allgemeine Optimierungsaufgaben mit nichtlinearen Nebenbedingungen empfohlen [17, S. 529]. Nach einer kurzen Einführung in die SQP in Abschnitt 2 werden in Abschnitt 3 exemplarisch die Modellparameter einer Regressionsgeraden unter Berücksichtigung verschiedener Zielfunktionen und Koordinatendarstellungen bestimmt. Die hierbei abgeleiteten Ergebnisse werden verwendet, um die Gültigkeit der zugesprochenen Eigenschaften der orthogonalen Regression zu eruieren. Abschnitt 4 fasst die Ergebnisse dieser Arbeit zusammen und gibt Hinweise zum Einsatz in der Metrologie.

\section{Parameterschätzung}

In der numerischen Optimierung zählt die sequentielle quadratische Programmierung zu den wichtigsten und effizientesten Verfahren zum Lösen von allgemeinen Optimierungsaufgaben mit nichtlinearen Nebenbedingungen
[3, S. 234]. Die SQP ist kein eigenständiges Verfahren. Vielmehr existiert eine ganze Reihe von Verfahren, die für unterschiedliche Anwendungsbereiche optimiert sind. Die folgende Ausführung beschränkt sich daher auf das Prinzip der SQP-Verfahren. Eine detaillierte Darstellung kann der Standardliteratur zur numerischen Optimierung entnommen werden $[3,17]$.

Das $\mathrm{zu}$ lösende Optimierungsproblem ist gegeben durch

$$
\min \Omega(\mathbf{u})
$$

unter der Nebenbedingung

$$
\boldsymbol{f}(\mathbf{u})=\mathbf{0},
$$

worin $\Omega$ die Zielfunktion symbolisiert, und $\mathbf{u}$ den Vektor der festen aber unbekannten Modellparameter repräsentiert. Sowohl die Zielfunktion $\Omega$ als auch die Nebenbedingung $\boldsymbol{f}$ seien glatte Funktionen. Das Lösen dieses Optimierungsproblems erfolgt mittels der Lagrange-Funktion, die unter Berücksichtigung der Lagrange-Multiplikatoren $\boldsymbol{\lambda}$ Zielfunktion und Nebenbedingung miteinander verknüpft [17, S. 530], d.h.,

$$
\mathcal{L}=\frac{1}{2} \Omega+\boldsymbol{\lambda}^{\mathrm{T}} \boldsymbol{f} .
$$

In diesem Beitrag wird davon ausgegangen, dass neben dem eindeutigen Minimum kein Maximum oder Sattelpunkt existiert. Die notwendige und hinreichende Optimalitätsbedingung an der Stelle $\left[\begin{array}{ll}\hat{\mathbf{u}}^{\mathrm{T}} & \hat{\boldsymbol{\lambda}}^{\mathrm{T}}\end{array}\right]$ ist somit erfüllt, wenn der Gradient der Lagrange-Funktion Null ist [12], d. h.,

$$
\begin{aligned}
& \nabla_{\mathbf{u}} \mathcal{L}(\hat{\mathbf{u}}, \hat{\boldsymbol{\lambda}})=\frac{1}{2} \nabla \Omega+\mathbf{J}^{\mathrm{T}} \hat{\boldsymbol{\lambda}}=\mathbf{0}, \\
& \nabla_{\boldsymbol{\lambda}} \mathcal{L}(\hat{\mathbf{u}}, \hat{\boldsymbol{\lambda}})=\boldsymbol{f}=\mathbf{0} .
\end{aligned}
$$

Hierin symbolisieren $\mathbf{J}$ die Jacobimatrix der Nebenbedingung und $\nabla \Omega$ den Gradienten der Zielfunktion.

Eine Möglichkeit, die unbekannten Modellparameter und Lagrange-Multiplikatoren zu bestimmen, ist die Anwendung des Newton-Verfahrens auf Gl. (3). Die Parameterschätzung erfolgt hierbei iterativ, sodass geeignete Startwerte $\mathbf{u}^{k=0}, \lambda^{k=0}$ bereitzustellen sind. Die zu lösende Newton-Gleichung des $k$-ten Iterationsschrittes liefert die neuen Iterierten $\hat{\mathbf{u}}=\mathbf{u}^{k}+\Delta \mathbf{u}$ und ist gegeben durch [17, S. 532]

$$
\left[\begin{array}{cc}
\nabla_{\mathbf{u u}}^{2} \mathcal{L} & \mathbf{J}^{\mathrm{T}} \\
\mathbf{J} & \mathbf{0}
\end{array}\right]\left[\begin{array}{c}
\Delta \mathbf{u} \\
\hat{\boldsymbol{\lambda}}
\end{array}\right]=-\left[\begin{array}{c}
\frac{1}{2} \nabla \Omega \\
\boldsymbol{f}
\end{array}\right]
$$

worin

$$
\nabla_{\mathbf{u u}}^{2} \mathcal{L}=\frac{1}{2} \nabla^{2} \Omega+\sum_{i=1} \lambda^{(i)} \mathbf{H}^{(i)}
$$


die Hessematrix der Lagrange-Funktion ist und $\nabla^{2} \Omega$ bzw. $\mathbf{H}^{(i)}$ die Hessematrizen der Zielfunktion bzw. der $i$-ten Nebenbedingung an der aktuellen Entwicklungsstelle darstellen. Die SQP-Verfahren minimieren somit in jedem Iterationsschritt das quadratische Teilproblem

$$
\min \frac{1}{2} \nabla^{\mathrm{T}} \Omega \Delta \mathbf{u}+\frac{1}{2} \Delta \mathbf{u}^{\mathrm{T}} \nabla_{\mathbf{u u}}^{2} \mathcal{L} \Delta \mathbf{u}
$$

unter der Nebenbedingung

$$
\mathbf{J} \Delta \mathbf{u}+\boldsymbol{f}=\mathbf{0} .
$$

Um den numerischen Aufwand zu reduzieren, wird in der praktischen Anwendung häufig auf das explizite Aufstellen der Hessematrizen in Gl. (4) verzichtet und i. A. auf das Quasi-Newton-Verfahren zurückgegriffen. Ein Überblick über mögliche Verfahren findet sich in $[3,17]$.

\section{Regressionsgerade}

Aufgrund der hohen Anschaulichkeit und in Anlehnung an die Untersuchungen von Kolaczia [9] wird nachfolgend die Gültigkeit für einige der zugesprochenen Eigenschaften der orthogonalen Regression am Beispiel einer Regressionsgeraden untersucht. Zur Parametrierung einer Geraden in der Ebene existieren eine Reihe von Darstellungen [2, Kap. 3.5.2.4]. Eine gebräuchliche Darstellung ist die Punkt-Richtung-Gleichung

$$
y_{i}=y_{0}+a\left(x_{i}-x_{0}\right)
$$

worin $\mathbf{P}_{0}^{\mathrm{T}}=\left(\begin{array}{ll}x_{0} & y_{0}\end{array}\right)$ die Koordinaten des Aufpunktes sind und $a$ die Steigung der Geraden darstellt. Weiterhin ist $\mathbf{P}_{i}^{\mathrm{T}}=\left(\begin{array}{ll}x_{i} & y_{i}\end{array}\right)$ ein beliebiger Punkt der Geraden. Aus der Substitution von $b=y_{0}-a x_{0}$ in Gl. (6) resultiert die kompakte Darstellung

$$
y_{i}=a x_{i}+b,
$$

worin $b$ den Schnittpunkt mit der $y$-Achse beschreibt.

Für $n$ Punkte enthält der Vektor der Modellparameter $\mathbf{u}$ neben dem Anstieg $a$ und dem $y$-Achsenabschnitt $b$ zusätzlich auch die Beobachtungsverbesserungen $\mathbf{v}$ der $2 n$ Koordinatenkomponenten und lautet

$$
\mathbf{u}^{\mathrm{T}}=\left(\begin{array}{llllllll}
a & b & v_{x_{1}} & \cdots & v_{x_{n}} & v_{y_{1}} & \cdots & v_{y_{n}}
\end{array}\right) .
$$

Die Nebenbedingung in Gl. (1b) ergibt sich unter Berücksichtigung von $\mathbf{v} \mathrm{zu}$

$$
0=a\left(x_{i}+v_{x_{i}}\right)+b-\left(y_{i}+v_{y_{i}}\right) .
$$

Entsprechend dem Prinzip der Methode der kleinsten (gewichteten) Verbesserungsquadratsumme lautet die Zielfunktion

$$
\Omega=\mathbf{u}^{\mathrm{T}} \mathbf{W} \mathbf{u}=\mathbf{v}^{\mathrm{T}} \mathbf{W}_{x y} \mathbf{v}
$$

worin

$$
\mathbf{W}=\left[\begin{array}{cc}
\mathbf{0} & \mathbf{0} \\
\mathbf{0} & \mathbf{W}_{x y}
\end{array}\right]
$$

die Extraktions- und Gewichtsmatrix der zu berücksichtigen Modellparameter in Gl. (1a) ist. Da das Minimum i. A. nur für $\mathbf{v}$ gefordert wird, ist hier lediglich die korrespondierende Matrix $\mathbf{W}_{x y} \mathrm{zu}$ spezifizieren.

Die Gewichtung steuert den Einfluss der Beobachtungen auf die Schätzung, wobei höhere Gewichte einen größeren Einfluss ausüben als kleinere. Insbesondere bei heterogenen Beobachtungen bietet sich eine Gewichtung an, die präzisere Beobachtungen stärker berücksichtigt. Eine geeignete Wahl für die Gewichtsmatrix ergibt sich aus der inversen Dispersionsmatrix der Beobachtungen [7, S. 104]. Beobachtungen mit kleinen Varianzen erhalten gegenüber Beobachtungen mit größeren Varianzen hierdurch ein höheres Gewicht. Im Gegenzug bedeutet eine Gleichgewichtung stochastische Isotropie des Beobachtungsmaterials. Hinweise für eine sachgerechte Abschätzung von Messunsicherheiten und zur Bildung der Dispersionsmatrix finden sich in $[11,4]$.

Zum Bilden der Newton-Gleichung in Gl. (4) sind der Gradient der Zielfunktion,

$$
\nabla \Omega=2 \mathbf{W u},
$$

sowie die Jacobimatrix der Nebenbedingung,

$$
\mathbf{J}=\left(\begin{array}{cccccccc}
x_{1}+v_{x_{1}}^{k} & 1 & a^{k} & \cdots & 0 & -1 & \cdots & 0 \\
\vdots & \vdots & \vdots & \ddots & \vdots & \vdots & \ddots & \vdots \\
x_{n}+v_{x_{n}}^{k} & 1 & 0 & \cdots & a^{k} & 0 & \cdots & -1
\end{array}\right),
$$

an der aktuellen Entwicklungsstelle $\mathbf{u}^{k} \mathrm{zu}$ bilden. Die Hessematrix der Zielfunktion lautet

$$
\nabla^{2} \Omega=2 \mathbf{W} .
$$

Die Hessematrizen der $n$ Nebenbedingung sind dünn besetzt. Sie besitzen jeweils an der mit $v_{x_{i}}$ korrespondierenden Zeile bzw. Spalte eine Eins in der ersten Spalte bzw. Zeile, d.h.,

$$
\mathbf{H}^{(i)}=\left(\begin{array}{ccccc}
0 & 0 & 1_{i} & 0 & \ldots \\
0 & 0 & 0 & 0 & \ldots \\
1_{i} & 0 & 0 & 0 & \ldots \\
0 & 0 & 0 & \ddots & \ldots \\
\vdots & \vdots & \vdots & \vdots & \ddots
\end{array}\right) .
$$


Tab. 1: Kartesische Koordinaten zur Schätzung der Modellparameter einer Regressionsgeraden in der Ebene.

\begin{tabular}{lrrrrr}
\hline Nr. & $\mathbf{P}_{\mathbf{1}}$ & $\mathbf{P}_{\mathbf{2}}$ & $\mathbf{P}_{\mathbf{3}}$ & $\mathbf{P}_{\mathbf{4}}$ & $\mathbf{P}_{\mathbf{5}}$ \\
\hline$x$ & 0,20 & 0,33 & 0,60 & 0,67 & 0,80 \\
$y$ & 0,47 & 0,27 & 0,33 & 0,07 & 0,13 \\
\hline
\end{tabular}

Das Iterationsverfahren wird solange wiederholt, bis die Optimalitätsbedingung in Gl. (3) erfüllt ist, wobei die Iterierten des vorangegangenen Berechnungsschrittes als verbesserte Startlösung im darauffolgenden Berechnungsschritt verwendet werden.

Zur Schätzung einer Regressionsgeraden liegen die in Tabelle 1 gegebenen $n=5$ fiktiven kartesischen Koordinaten vor. Die $x$ - und $y$-Komponenten dieser Punkte seien jeweils unabhängige und identisch verteilte Zufallsvariablen, sodass die Gewichtsmatrix durch

$$
\mathbf{W}_{x y}=\left[\begin{array}{cc}
w_{x} \mathbf{I} & \mathbf{0} \\
\mathbf{0} & w_{y} \mathbf{I}
\end{array}\right]
$$

beschrieben wird. Hierin ist I die Einheitsmatrix und $w_{x}$ bzw. $w_{y}$ die einheitlichen Gewichtungen für die $x$ - bzw. $y$-Komponente.

Im Folgenden soll geprüft werden, welche Rolle die Gewichtung auf die Schätzung hat. Ferner wird untersucht, ob die Ergebnisse bzgl. einer Koordinatentransformation invariant sind und wie sich ein Wechsel der Koordinatendarstellung auswirkt.

\subsection{Einfluss der Gewichtung}

Um den Einfluss der Gewichtung auf die Schätzung zu demonstrieren, wurden drei unterschiedliche Gewichtsverhältnisse bei der Bestimmung der Regressionsgeraden gewählt.

Abbildung 1 zeigt neben den fünf Punkten (siehe Tabelle 1) die resultierenden Regressionsgeraden für drei unterschiedlich gewählte Gewichtungen, $w_{x} \gg w_{y}(1 \mathrm{a}), w_{x}=$ $w_{y}(1 \mathrm{~b})$ und $w_{x} \ll w_{y}$ (1c). Weiterhin sind die geschätzten Verbesserungsvektoren der einzelnen Schätzungen durch schwarze Pfeile eingezeichnet. Numerische Werte für die geschätzten Modellparameter sind in Tabelle 2 zusammengestellt.

Für $w_{x} \gg w_{y}$ bzw. $w_{x} \ll w_{y}$ gehen die geschätzten Verbesserungen $v_{x}$ bzw. $v_{y}$ gegen Null, siehe Tabelle 2 . Für entsprechend große Gewichtsverhältnisse gehen diese Lösungen somit in eine Ausgleichung über, die nur die horizontalen bzw. vertikalen Abstände minimiert. Die Gewichtsmatrix entspricht der inversen Dispersionsmatrix
Tab. 2: Zusammenstellung der geschätzten Modellparameter für drei unterschiedlich gewählte Gewichtsverhältnisse.

\begin{tabular}{lrrr}
\hline & $w_{\boldsymbol{x}} \gg w_{\boldsymbol{y}}$ & $\boldsymbol{w}_{\boldsymbol{x}}=\boldsymbol{w}_{\boldsymbol{y}}$ & $\boldsymbol{w}_{\boldsymbol{x}} \ll \boldsymbol{w}_{\boldsymbol{y}}$ \\
\hline$a$ & $-0,5224$ & $-0,5860$ & $-0,7938$ \\
$b$ & 0,5256 & 0,5587 & 0,6668 \\
$v_{x_{1}}$ & 0,0000 & $-0,0124$ & 0,0479 \\
$v_{x_{2}}$ & 0,0000 & 0,0416 & 0,1698 \\
$v_{x_{3}}$ & 0,0000 & $-0,0536$ & $-0,1757$ \\
$v_{x_{4}}$ & 0,0000 & 0,0419 & 0,0818 \\
$v_{x_{5}}$ & 0,0000 & $-0,0175$ & $-0,1238$ \\
$v_{y_{1}}$ & $-0,0488$ & $-0,0212$ & 0,0000 \\
$v_{y_{2}}$ & 0,0833 & 0,0710 & 0,0000 \\
$v_{y_{3}}$ & $-0,1178$ & $-0,0915$ & 0,0000 \\
$v_{y_{4}}$ & 0,1056 & 0,0715 & 0,0000 \\
$v_{y_{5}}$ & $-0,0223$ & $-0,0298$ & 0,0000 \\
\hline
\end{tabular}

der Beobachtungen. Beobachtungen mit einer kleinen Varianz bzw. einem großen Gewicht weisen somit geringere Verbesserungen auf und beeinflussen die Schätzung stärker als Beobachtungen mit größeren Varianzen. Wird hingegen stochastische Isotropie, $d . h$. identische Gewichte $w_{x}=w_{y}$ gewählt, so stehen die einzelnen Verbesserungsvektoren der Punkte senkrecht auf der geschätzten Geraden. Diese Lösung entspricht einer orthogonalen Regression, welche somit als Spezialfall eines allgemeinen Optimierungsproblems aufgefasst werden darf.

\subsection{Koordinatentransformation}

Unter einer Koordinatentransformation wird in diesem Beitrag eine Verschiebung, Drehung oder Skalierung des Koordinatensystems verstanden. Für die orthogonale Regression gibt Ahn [1, Kap. 1.2.2] an, dass die Schätzungen durch eine Transformation der Koordinaten nicht beeinflusst wird. Intrinsische Modellparameter wie bspw. der Radius eines Kreises bleiben somit invariant. Kolaczia [9] weist andererseits daraufhin, dass eine Skalierung zu unterschiedlichen Ergebnissen führt und somit die Ergebnisse nicht mehr unabhängig von den gewählten Maßeinheiten sind. Im Folgenden werden beide Aussagen untersucht. Zwei Schätzungen werden hierbei als äquivalent angesehen, wenn deren (gewichtete) Verbesserungsquadratsummen identisch und somit unabhängig von der Koordinatentransformation sind.

\subsubsection{Verschiebung}

Eine Verschiebung der Koordinaten um $t_{x}$ bzw. $t_{y}$ führt mit $x_{0}+t_{x}$ und $y_{0}+t_{y} z u$ einer Änderung in den Koordinaten des 


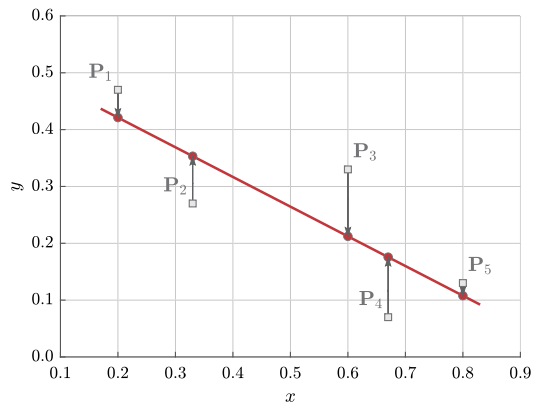

a) $w_{x} \gg w_{y}$

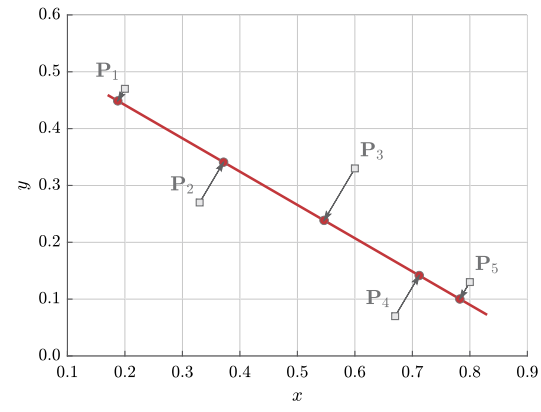

b) $w_{x}=w_{y}$

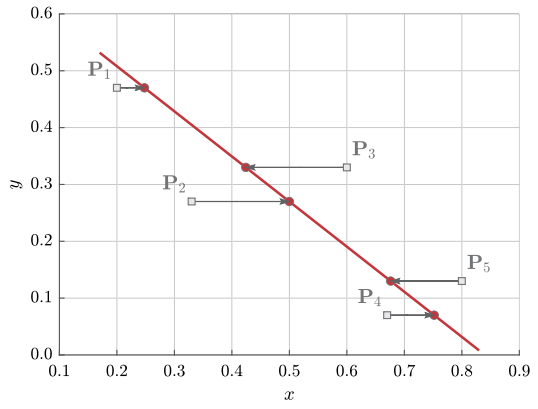

c) $w_{x} \ll w_{y}$

Abb. 1: Resultierende Regressionsgeraden bei unterschiedlich gewählten Gewichtsverhältnissen: (a) $w_{x} \gg w_{y}$, (b) $w_{x}=w_{y}$ und (c) $w_{x} \ll w_{y}$. Die originären Koordinaten sind durch graue Quadrate und die jeweils verbesserten Koordinaten der einzelnen Schätzungen sind durch rote Kreise dargestellt. Schwarze Pfeile symbolisieren die geschätzten Verbesserungsvektoren. Für $w_{x}=w_{y}$ stehen diese Verbesserungsvektoren senkrecht auf der geschätzten Geraden.

Aufpunktes in Gl. (6). Die (gewichtete) Verbesserungsquadratsumme bleibt jedoch unverändert. Wie man sich leicht an Abbildung 1 veranschaulichen kann, trifft dies auf jedes Optimierungsproblem zu und ist somit keine exklusive Eigenschaft der orthogonalen Regression. Es handelt sich um den Trivialfall, sodass sich eine weitere Untersuchung erübrigt.

\subsubsection{Drehung}

Eine Drehung wird allgemein durch eine orthogonale Matrix $\mathbf{R}$ parametriert, für die $\mathbf{R R}^{\mathrm{T}}=\mathbf{R}^{\mathrm{T}} \mathbf{R}=\mathbf{I}$ und $\operatorname{det} \mathbf{R}=+1$ gilt [16]. Für eine Drehung in der Ebene um den Winkel $\alpha$ ergibt sich die Rotationsmatrix zu

$$
\mathbf{R}=\left(\begin{array}{rr}
\cos \alpha & -\sin \alpha \\
\sin \alpha & \cos \alpha
\end{array}\right)
$$

Eine Koordinatentransformation, die ausschließlich eine Drehung um den Koordinatenursprung beschreibt, ist gegeben durch

$$
\mathbf{L}^{\prime}=(\mathbf{R} \otimes \mathbf{I}) \mathbf{L},
$$

worin

$$
\mathbf{L}^{\mathrm{T}}=\left(\begin{array}{llllllll}
x_{1} & x_{2} & \ldots & x_{n} & y_{1} & y_{2} & \ldots & y_{n}
\end{array}\right)
$$

und $\mathbf{L}^{\prime}$ die Vektoren der originären bzw. der rotierten Koordinaten sind und $\otimes$ das Kronecker-Produkt symbolisiert [2, S. 258]. Da diese Transformation sowohl auf die originären Koordinaten als auch auf die verbesserten Koordinaten angewendet werden darf, ergibt sich für den Verbesserungsvektor die wichtige Beziehung

$$
\begin{aligned}
\mathbf{v}^{\prime} & =(\mathbf{R} \otimes \mathbf{I})(\mathbf{L}+\mathbf{v}-\mathbf{L}) \\
& =(\mathbf{R} \otimes \mathbf{I}) \mathbf{v} .
\end{aligned}
$$

Mittels der linearen Transformation in Gl. (14) ist auch die Gewichtsmatrix zu transformieren, da diese ebenfalls vom gewählten Koordinatensystem bzw. Datum abhängt. Die transformierte Gewichtsmatrix lautet [7, S. 100]

$$
\mathbf{W}_{x y}^{\prime}=\left((\mathbf{R} \otimes \mathbf{I}) \mathbf{W}_{x y}^{-1}(\mathbf{R} \otimes \mathbf{I})^{\mathrm{T}}\right)^{-1} .
$$

Für die Zielfunktion in Gl. (10) folgt somit unmittelbar

$$
\begin{aligned}
\Omega^{\prime} & =\mathbf{v}^{\prime \mathrm{T}} \mathbf{W}_{x y}^{\prime} \mathbf{v}^{\prime} \\
& =\mathbf{v}^{\mathrm{T}}(\mathbf{R} \otimes \mathbf{I})^{\mathrm{T}}\left((\mathbf{R} \otimes \mathbf{I}) \mathbf{W}_{x y}^{-1}(\mathbf{R} \otimes \mathbf{I})^{\mathrm{T}}\right)^{-1}(\mathbf{R} \otimes \mathbf{I}) \mathbf{v} \\
& =\mathbf{v}^{\mathrm{T}}(\mathbf{R} \otimes \mathbf{I})^{\mathrm{T}}(\mathbf{R} \otimes \mathbf{I}) \mathbf{W}_{x y}(\mathbf{R} \otimes \mathbf{I})^{\mathrm{T}}(\mathbf{R} \otimes \mathbf{I}) \mathbf{v} \\
& =\mathbf{v}^{\mathrm{T}} \mathbf{W}_{x y} \mathbf{v} \\
& =\Omega .
\end{aligned}
$$

Beide Schätzungen liefern identische (gewichtete) Verbesserungsquadratsummen und sind somit äquivalent. Die Modellparameter mögen aufgrund des geänderten Koordinatensystems differieren, lassen sich jedoch durch eine geeignete Transformation ineinander überführen. Es handelt sich somit um keine exklusive Eigenschaft der orthogonalen Regression. Für die rotierte Gerade ergeben sich Anstieg und $y$-Achsenabschnitt aus

$$
a^{\prime}=\tan (\alpha+\arctan a)
$$

bzw.

$$
b^{\prime}=b\left(\cos \alpha+a^{\prime} \sin \alpha\right)
$$

Während sich die Vektorkomponenten durch Multiplikation mit einer orthogonalen Matrix ändern, bleibt die Länge des Vektors unverändert. Tabelle 3 stellt die resultierenden Verbesserungsvektoren sowie deren Länge für 
Tab. 3: Gegenüberstellung der geschätzten Verbesserungsvektoren sowie deren Länge für die Regressionsgerade vor und nach einer Drehung (Gewichtung $w_{x}=w_{y}$ ). Die Drehung wurde so gewählt, dass der Anstieg der gedrehten Geraden $a^{\prime}=-1$ ist.

\begin{tabular}{lrrrrrr}
\hline Nr. & $\boldsymbol{v}_{\boldsymbol{x}}$ & $\boldsymbol{v}_{\boldsymbol{y}}$ & $|\mathbf{v}|$ & $\boldsymbol{v}_{\boldsymbol{x}}^{\prime}$ & $\boldsymbol{v}_{\boldsymbol{y}}^{\prime}$ & $\left|\mathbf{v}^{\prime}\right|$ \\
\hline $\mathbf{P}_{1}$ & $-0,0124$ & $-0,0212$ & 0,0246 & $-0,0174$ & $-0,0174$ & 0,0246 \\
$\mathbf{P}_{2}$ & 0,0416 & 0,0710 & 0,0823 & 0,0582 & 0,0582 & 0,0823 \\
$\mathbf{P}_{3}$ & $-0,0536$ & $-0,0915$ & 0,1060 & $-0,0750$ & $-0,0750$ & 0,1060 \\
$\mathbf{P}_{4}$ & 0,0419 & 0,0715 & 0,0829 & 0,0586 & 0,0586 & 0,0829 \\
$\mathbf{P}_{5}$ & $-0,0175$ & $-0,0298$ & 0,0346 & $-0,0244$ & $-0,0244$ & 0,0346 \\
\hline
\end{tabular}

die Regressionsgerade vor und nach einer Drehung gegenüber. Der Drehwinkel $\alpha$ wurde hierbei so gewählt, dass die Steigung der gedrehten Geraden $-45^{\circ}$ beträgt. Dies entspricht einem Anstieg von $a^{\prime}=-1$. Für die Komponenten der Verbesserungsvektoren ergibt sich hierdurch bei identischer Gewichtung $v_{x_{i}}^{\prime}=v_{y_{i}}^{\prime}$. Da die mittels Gl. (14) ermittelten Vektorkomponenten vom gewählten Datum abhängen, erscheinen diese für eine objektive Bewertung ungeeignet.

\subsubsection{Skalierung}

Eine Skalierung der Beobachtungen wird nötig, wenn bspw. zwischen Maßeinheiten umzurechnen ist. Sie lässt sich allgemein durch eine reguläre Diagonalmatrix $\mathbf{S}$ darstellen, welche auf der Hauptdiagonalen die Skalierungsparameter enthält. Die lineare Koordinatentransformation, die ausschließlich eine Skalierung berücksichtigt, ist gegeben durch

$$
\mathbf{L}^{\prime \prime}=\mathbf{S} \mathbf{L},
$$

worin $\mathbf{L}$ und $\mathbf{L}^{\prime \prime}$ die Vektoren der originären bzw. der skalierten Koordinaten sind. Verbesserungen und Gewichtsmatrix lauten

$$
\begin{aligned}
\mathbf{v}^{\prime \prime} & =\mathbf{S}(\mathbf{L}+\mathbf{v}-\mathbf{L}) \\
& =\mathbf{S} \mathbf{v}
\end{aligned}
$$

bzw.

$$
\mathbf{W}_{x y}^{\prime \prime}=\left(\mathbf{S W}_{x y}^{-1} \mathbf{s}\right)^{-1} .
$$

Für die Zielfunktion in Gl. (10) folgt

$$
\begin{aligned}
\Omega^{\prime \prime} & =\mathbf{v}^{\prime \prime \mathrm{T}} \mathbf{W}_{x y}^{\prime \prime} \mathbf{v}^{\prime \prime} \\
& =\mathbf{v}^{\mathrm{T}} \mathbf{S}\left(\mathbf{S W}_{x y}^{-1} \mathbf{S}\right)^{-1} \mathbf{S} \mathbf{v} \\
& =\mathbf{v}^{\mathrm{T}} \mathbf{S} \mathbf{S}^{-1} \mathbf{W}_{x y} \mathbf{S}^{-1} \mathbf{S} \mathbf{v} \\
& =\mathbf{v}^{\mathrm{T}} \mathbf{W}_{x y} \mathbf{v} \\
& =\Omega .
\end{aligned}
$$

Beide Schätzungen liefern identische (gewichtete) Verbesserungsquadratsummen und sind somit äquivalent. Durch die Skalierung ändern sich $u$.U. die geschätzten Modellparameter, diese können jedoch durch eine geeignete Transformation ineinander überführt werden. Seien $s_{x}$ und $s_{y}$ die Skalierungen der $x$ - bzw. $y$-Komponente, so resultieren Anstieg und $y$-Achsenabschnitt aus

$$
a^{\prime \prime}=\frac{s_{y}}{s_{x}} a
$$

bzw.

$$
b^{\prime \prime}=s_{y} b .
$$

Standen die Verbesserungsvektoren vor der Transformation senkrecht auf der Geraden, so stehen die skalierten Vektoren aufgrund von Gl. (17) nach der Transformation nicht mehr senkrecht auf der Geraden, sofern es sich bei $\mathbf{S}$ nicht um eine einheitlich skalierte Einheitsmatrix handelt.

Unabhängig von der Orientierung der Verbesserungsvektoren bleibt die zugewiesene physische oder geometrische Bedeutung der Modellparameter erhalten und eine Umrechnung zwischen Maßeinheiten ist problemlos möglich. Es handelt sich somit um keine exklusive Eigenschaft der orthogonalen Regression. $\mathrm{Zu}$ beachten ist, dass der Wechsel der Maßeinheit bzw. die Skalierung nicht nur für die Messwerte selbst, sondern auch für deren Dispersion durchzuführen ist. Bei Missachtung werden unterschiedliche Zielfunktionen minimiert, sodass die Schätzungen nicht mehr äquivalent zueinander sind, wie Kolaczia [9] zeigt. Weiterhin zeigt das Beispiel der Skalierung anschaulich, dass jedes allgemeine Optimierungsproblem durch eine geeignete Modifikation in das spezielle Problem einer orthogonalen Regression überführbar ist.

\subsection{Koordinatendarstellung}

In einigen Anwendungen in der Koordinatenmesstechnik werden anstelle von kartesischen Koordinaten die korrespondierenden polaren Messelemente registriert, 
bspw. bei Verwendung eines Lasertrackers [5]. Eine Umrechnung zwischen beiden Koordinatendarstellungen ist widerspruchsfrei möglich. In der Ebene lautet die Umformung von Polarkoordinaten in kartesische Koordinaten

$$
\begin{aligned}
& x_{i}=r_{i} \cos \phi_{i}, \\
& y_{i}=r_{i} \sin \phi_{i} .
\end{aligned}
$$

Die Umformung von kartesischen Koordinaten in Polarkoordinaten ist gegeben durch

$$
\begin{aligned}
\phi_{i} & =\arctan \frac{y_{i}}{x_{i}}, \\
r_{i} & =\sqrt{x_{i}^{2}+y_{i}^{2}} .
\end{aligned}
$$

Hierin bezeichnet $r_{i}$ die Strecke und $\phi_{i}$ den Richtungswinkel.

In diesem Abschnitt wird untersucht, ob die gewählte Koordinatendarstellung die Schätzung beeinflusst, oder ob die Ergebnisse unabhängig hiervon sind. Einerseits geben Holst et al. [6] an, dass eine orthogonale Regression nur mit kartesischen Koordinaten durchführbar sei. Andererseits zeigen Koch \& Kargoll [8], dass die Ausgleichungsergebnisse unabhängig von der Wahl der Koordinatendarstellung sind. Eine Umrechnung wäre demnach unnötig.

Um zu prüfen, inwieweit sich eine gewählte Koordinatendarstellung auf die Schätzung auswirkt, werden die Koordinaten aus Tabelle 1 mittels Gl. (21) in ihre polare Darstellung umgeformt. Weiterhin ergibt die Substitution der Gl. (20) in Gl. (7) die Regressionsgerade mit Polarkoordinaten, d.h.,

$$
r_{i} \sin \phi_{i}=a\left(r_{i} \cos \phi_{i}\right)+b .
$$

Unter Berücksichtigung der polaren Verbesserungen lautet die in Gl. (1b) zu berücksichtigende Nebenbedingung

$$
\begin{aligned}
0= & a\left(r_{i}+v_{r_{i}}\right) \cos \left(\phi_{i}+v_{\phi_{i}}\right)+ \\
& +b-\left(r_{i}+v_{r_{i}}\right) \sin \left(\phi_{i}+v_{\phi_{i}}\right) .
\end{aligned}
$$

Gemäß dem Prinzip der Methode der kleinsten Quadrate wird Gl. (10) als Zielfunktion verwendet. Der Verbesserungsvektor enthält hierbei jedoch die polaren Verbesserungen $\mathbf{v}^{\mathrm{T}}=\left(\begin{array}{llllll}v_{\phi_{1}} & \ldots & v_{\phi_{n}} & v_{r_{1}} & \ldots & v_{r_{n}}\end{array}\right)$ und $\mathbf{W}_{x y}$ wird sinngemäß durch $\mathbf{W}_{\phi r}$ ersetzt. Im Abschnitt 3.1 konnte bereits gezeigt werden, dass eine orthogonale Regression durch eine einheitliche Gewichtung realisiert werden kann. Als Gewichtung wird daher $\mathbf{W}_{\phi r}=\mathbf{I}$ verwendet.

Abbildung 2 zeigt die resultierende Regressionsgerade mit den geschätzten Formparametern $a=-0,634$ und $b=0,584$ in kartesischer Darstellung. Numerische Werte für die Verbesserungen sind in Tabelle 4 zusammengestellt. Gut zu erkennen ist, dass die Verbesserungsvektoren nicht senkrecht auf dieser Geraden stehen. Dass es sich

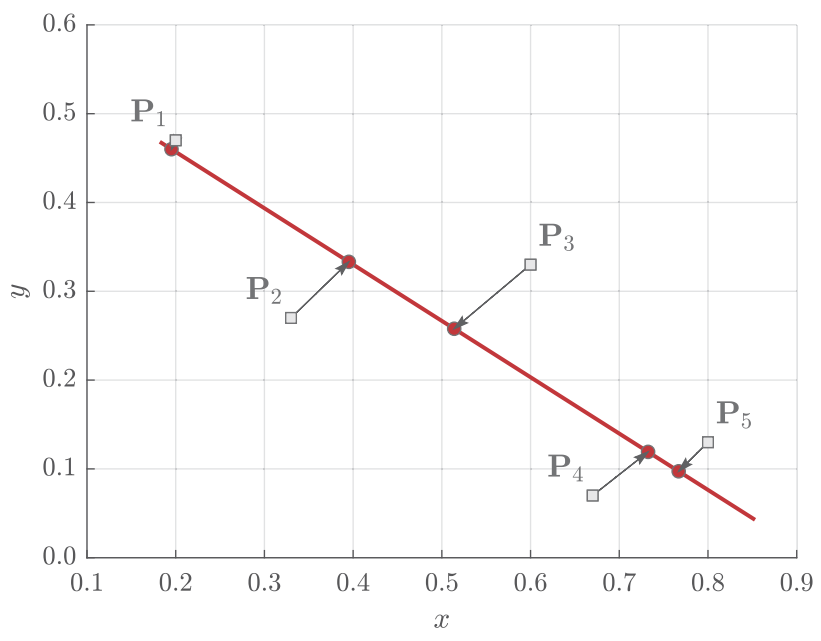

Abb. 2: Resultierende Regressionsgerade bei Verwendung von Polarkoordinaten. Für diese Darstellung wurden die polaren Elemente mittels Gl. (20) umgeformt. Graue Quadrate und rote Kreise entsprechen den originären bzw. verbesserten Koordinaten in kartesischer Darstellung. Schwarze Pfeile symbolisieren die jeweiligen Verbesserungsvektoren, die nicht senkrecht auf der geschätzten Geraden stehen.

Tab. 4: Verbesserungsvektoren in kartesischer Darstellung für die Regressionsgerade bei Verwendung von Polarkoordinaten.

\begin{tabular}{rrrrrr}
\hline & $\mathbf{P}_{\mathbf{1}}$ & $\mathbf{P}_{\mathbf{2}}$ & $\mathbf{P}_{\mathbf{3}}$ & $\mathbf{P}_{\mathbf{4}}$ & $\mathbf{P}_{\mathbf{5}}$ \\
\hline$v_{x}$ & $-0,0048$ & 0,0652 & $-0,0861$ & 0,0625 & $-0,0330$ \\
$v_{y}$ & $-0,0100$ & 0,0632 & $-0,0722$ & 0,0492 & $-0,0327$ \\
$|\mathbf{v}|$ & 0,0111 & 0,0907 & 0,1123 & 0,0795 & 0,0465 \\
\hline
\end{tabular}

dennoch um eine orthogonale Regression handelt, kann Abbildung 3 leicht entnommen werden.

Abbildung 3 zeigt die identische Regressionsgerade in polarer Darstellung, die sich durch Umformung von Gl. (22) $\mathrm{zu}$

$$
r_{i}=f\left(\phi_{i}\right)=\frac{b}{\left(\sin \phi_{i}-a \cos \phi_{i}\right)}
$$

ergibt. Gut zu erkennen ist, dass in dieser Darstellung die Verbesserungsvektoren senkrecht auf der Regressionskurve stehen, sodass es sich um eine orthogonale Regression handelt. Eine orthogonale Regression ist folglich auch mit Polarkoordinaten möglich und beschränkt sich nicht auf die Verwendung kartesischer Koordinaten.

Die Abbildungen 2 und 3 zeigen identische Schätzungen in unterschiedlichen Koordinatendarstellungen. Besitzen die Formparameter $a$ und $b$ eine physische oder geometrische Bedeutung in der kartesischen Darstellung, so ist diese in der Polardarstellung nicht unmittelbar erkennbar - wenngleich die Verbesserungen hier orthogo- 


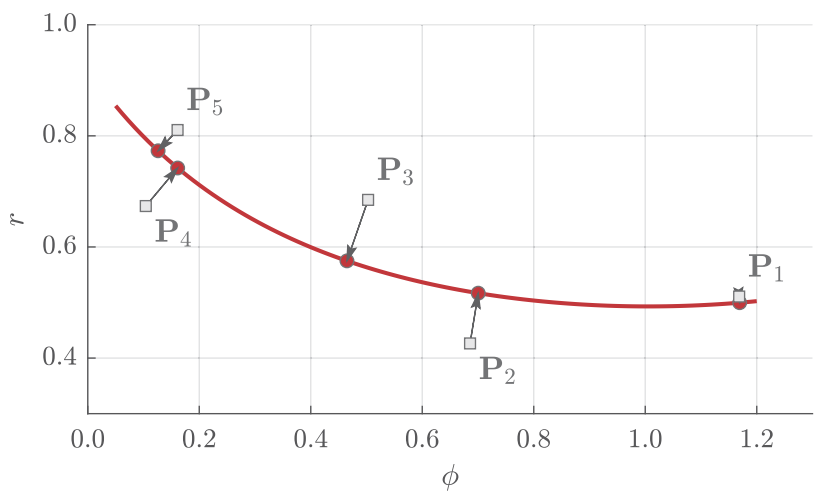

Abb. 3: Darstellung der geschätzten Regressionsgeraden in Abhängigkeit der polaren Elemente $\phi$ und $r$. Graue Quadrate und rote Kreise entsprechen den originären bzw. verbesserten Koordinaten in polarer Darstellung. Schwarze Pfeile symbolisieren die jeweiligen Verbesserungsvektoren, die senkrecht auf der geschätzten Funktion stehen.

nal sind. Die orthogonale Regression liefert somit keine Modellparameter, die eine physische oder geometrische Bedeutung aufweisen müssen. Dies ist auch nicht die Aufgabe des Schätzers. Vielmehr weist der Analyst durch eine entsprechende Modellbildung den hierfür gewählten Modellparametern der Schätzung eine physische oder geometrische Bedeutung zu.

Weiterhin veranschaulichen die Ergebnisse, dass diese nicht unabhängig von der Koordinatendarstellung sind, da jeweils unterschiedliche Zielfunktionen minimiert werden [13]. Koch \& Kargoll [8] zeigen, dass die Ergebnisse einer Schätzung nur dann unabhängig von der Koordinatendarstellung sind, wenn eine linearisierte Transformation verwendet wird. Die Umformungen nach Gl.(20) bzw. Gl. (21) sind nichtlinear, sodass diese Aussage nicht verallgemeinert werden darf. In der Praxis treten jedoch meist nur kleine Verbesserungen auf, sodass die Differenzen, die aus den unterschiedlich gewählten Koordinatendarstellungen resultieren, häufig vernachlässigbar sind.

Lösler [13] zeigt, dass identische Ergebnisse durch eine sachgerechte Modifikation der Zielfunktion gewonnen werden. Mit der Zielfunktion der polaren Verbesserungen

$$
\begin{aligned}
\Omega\left(\mathbf{v}_{\phi}, \mathbf{v}_{r}\right) & =\sum_{i=1}^{n}\left(\left(r_{i}+v_{r_{i}}\right) \cos \left(\phi_{i}+v_{\phi_{i}}\right)-r_{i} \cos \phi_{i}\right)^{2}+ \\
& +\sum_{i=1}^{n}\left(\left(r_{i}+v_{r_{i}}\right) \sin \left(\phi_{i}+v_{\phi_{i}}\right)-r_{i} \sin \phi_{i}\right)^{2}
\end{aligned}
$$

ergeben sich die Ergebnisse der orthogonalen Regression im kartesischen Datum. Entsprechend lassen sich die Ergebnisse der orthogonalen Regression im polaren Datum mit der Zielfunktion

$$
\begin{aligned}
\Omega\left(\mathbf{v}_{x}, \mathbf{v}_{y}\right) & =\sum_{i=1}^{n}\left(\arctan \frac{y_{i}+v_{y_{i}}}{x_{i}+v_{x_{i}}}-\arctan \frac{y_{i}}{x_{i}}\right)^{2}+ \\
& +\sum_{i=1}^{n}\left(\sqrt{\left(x_{i}+v_{x_{i}}\right)^{2}+\left(y_{i}+v_{y_{i}}\right)^{2}}-\sqrt{x_{i}^{2}+y_{i}^{2}}\right)^{2}
\end{aligned}
$$

replizieren, wenn kartesische Koordinaten verwendet werden. Ein vorheriger Wechsel der Koordinatendarstellung ist demnach unnötig.

Der Wechsel der Koordinatendarstellung ist ein weiteres anschauliches Beispiel dafür, dass jedes allgemeine Optimierungsproblem durch eine geeignete Modifikation in das spezielle Problem einer orthogonalen Regression überführbar ist, vgl. Abbildung 2 und 3. Ob die Verbesserungen senkrecht auf der Funktion stehen, hängt von der gewählten Modellbildung, d. h, vom funktionalen Modell, vom stochastischen Modell und von der gewählten Zielfunktion ab.

\section{Zusammenfassung}

In vielen Anwendungen wird zur Datenanalyse auf die orthogonale Regression zurückgegriffen und somit stochastische Isotropie der Beobachtungen unterstellt, bei der die zu minimierenden Verbesserungen senkrecht auf der Funktion stehen. Einer der zumeist aufgeführten Hauptgründe für den Einsatz dieses Verfahrens ist der hohe Anschauungsgrad. In diesem Beitrag wurde untersucht, ob ein Teil der zugesprochenen Eigenschaften der orthogonalen Regression exklusive Eigenschaften dieses Verfahrens sind oder ob es sich um grundlegende Eigenschaften eines allgemeinen Optimierungsproblems handelt.

Es wurde untersucht, ob die Modellparameter der orthogonalen Regression eine spezielle physische oder geometrische Bedeutung aufweisen, die Lösungen eines allgemeinen Optimierungsproblems nicht besitzen. Die Untersuchungen in Abschnitt 3 zeigen, dass die physische oder geometrische Bedeutung der Modellparameter nicht aus dem gewählten Optimierungsverfahren resultiert, sondern sich aus der gewählten Modellbildung, d.h. dem funktionalen Modell, dem stochastischen Modell und der gewählten Zielfunktion, ergibt. Ein gewähltes Optimierungsverfahren weist den Modellparametern grundsätzlich keine physische oder geometrische Bedeutung zu. Dies ist Aufgabe des Anwenders bei der Analyse und der Ergebnisinterpretation.

Im Abschnitt 3.2 wurde geprüft, ob die Ergebnisse eines allgemeinen Optimierungsproblems durch eine Koor- 
dinatentransformation beeinflusst werden. Denkbare Anwendungen sind das Zusammenführen von Bauteilen mit unterschiedlichen Objektkoordinatensystemen oder das Umrechnen von Maßeinheiten. Es konnte gezeigt werden, dass sich die Zielfunktion durch eine Koordinatentransformation nicht ändert, sodass die Ergebnisse äquivalent zueinander sind. Änderungen in den geschätzten Modellparametern lassen sich durch eine geeignete Transformation umkehren. Dies wurde exemplarisch für die Parameter der Geraden gezeigt. Standen die Verbesserungsvektoren vor der Transformation senkrecht auf der Funktion, so können diese in Abhängigkeit der gewählten Transformation jedoch nach der Transformation eine beliebige Orientierung annehmen.

Eine orthogonale Regression ist unabhängig von der Koordinatendarstellung der Beobachtungen und sowohl mit kartesischen Koordinaten als auch mit Polarkoordinaten durchführbar. Ob die kartesischen oder die polaren Verbesserungen senkrecht auf der geschätzten Funktion stehen, hängt vom funktionalen Modell, vom stochastischen Modell und von der gewählten Zielfunktion ab, wie die Ergebnisse in Abschnitt 3.2 zeigen.

Für die Datenanalyse wurde auf die sequentielle quadratische Programmierung zurückgegriffen. In der numerischen Optimierung wird die SQP insbesondere für allgemeine Optimierungsaufgaben mit nichtlinearen Nebenbedingungen empfohlen. In diesem Beitrag wurde die SQP zur Einordnung der Ergebnisse der orthogonalen Regression verwendet. Es konnte gezeigt werden, dass von den unendlich vielen Formulierungen eines Optimierungsproblems die orthogonale Regression lediglich ein spezielles beschreibt, siehe z. B. Abbildung 1. Weiterhin wurde in den Abschnitten 3.1 und 3.3 exemplarisch gezeigt, dass sich jedes allgemeine Optimierungsproblem durch eine geeignete Modellmodifikation in das spezielle Problem der orthogonalen Regression überführen lässt, welches äquivalente Ergebnisse liefert.

Die Motivation für diese Untersuchungen war die durch Kolaczia [9] aufgeworfene Frage, ob die orthogonale Regression für die Auswertung von Daten in der Metrologie geeignet ist. Die orthogonale Regression kann geeignet sein, da diese eine spezielle Formulierung eines allgemeinen Optimierungsproblems darstellt. Kolaczia [9] zeigt jedoch anschaulich am Beispiel einer Skalierung, dass ohne geeignete Maßnahmen die Ergebnisse der orthogonalen Regression widersprüchlich werden können. Die orthogonale Regression ist demnach kein Analysewerkzeug, das universell auf jede Problemstellung direkt anwendbar ist. Hierin wiederum liegt die Stärke eines allgemeinen Optimierungsverfahrens wie bspw. der in Abschnitt 2 vorgestellten sequentiellen quadratischen Programmierung.
Danksagung: Wir widmen diesen Beitrag Frau Prof. Dr.-Ing. Maria Hennes vom Geodätischen Institut des Karlsruher Instituts für Technologie.

\section{Literatur}

1. AнN, S. J. Least Squares Orthogonal Distance Fitting of Curves and Surfaces in Space. Lecture Notes in Computer Science, vol. 3151. Springer, Berlin, Heidelberg, 2004. doi: $10.1007 /$ b104017.

2. Bronshtein, I. N., Semendyayev, K. A., Musiol, G., Und Muehlig, H. Handbook of Mathematics, 5. Auflage. Springer, Berlin, Heidelberg, 2007. doi: 10.1007/978-3-540-72122-2.

3. GEIGER, C., UND KANZow, C. Theorie und Numerik restringierter Optimierungsaufgaben. Springer, Berlin, Heidelberg, 2002. doi: 10.1007/978-3-642-56004-0.

4. Hennes, M. Zum Umgang mit Unsicherheiten bei geodätischen Mess- und Auswerteverfahren. In 125. DVW-Seminar:

Qualitätssicherung geodätischer Mess- und Auswertverfahren, I. Neumann, V. Schwieger, und K. Fritzensmeier, Eds., DVW-Schriftenreihe, vol. 71. Wißner-Verlag, Augsburg, 2013, S. 25-46.

5. Hennes, M. Messmittel der Large Volume Metrology (LVM). In Handbuch der Geodäsie - Ingenieurgeodäsie, W. Freeden und R. Rummel, Eds., Springer Reference Naturwissenschaften. Springer, Berlin, Heidelberg, 2017, S. 347-370. doi: 10.1007/978-3-662-47188-3_26.

6. Holst, C., Zeimetz, P., Nothnagel, A., Schauerte, W., und KUHLMANN, H. Estimation of focal length variations of a 100-m radio telescope's main reflector by laser scanner measurements. Journal of Surveying Engineering 138, 3 (2012), 126-135. doi: 10.1061/(asce)su.1943-5428.0000082.

7. Косн, K.-R. Parameter Estimation and Hypothesis Testing in Linear Models. Springer, Berlin, Heidelberg, 1999. doi: 10.1007/978-3-662-03976-2.

8. KoCH, K.-R., UND KARGOLL, B. Outlier detection by the EM algorithm for laser scanning in rectangular and polar coordinate systems. Journal of Applied Geodesy 9, 3 (2015), 162-173. doi: 10.1515/jag-2015-0004.

9. KolaCZIA, W. Das Problem der linearen Ausgleichung im R2. tm - Technisches Messen 73, 11 (2006), 629-633. doi: 10.1524/teme.2006.73.11.629.

10. KRYSTEK, M. Ausgleichsgeraden in der Ebene. tm - Technisches Messen 71, 1 (2004), 19-23. doi: 10.1524/teme.2011.0118.

11. Krystek, M. Berechnung der Messunsicherheit - Grundlagen und Anleitung für die praktische Anwendung. Messwesen. DIN Deutsches Institut für Normung e. V., Beuth Verlag, Berlin, 2012.

12. Lenzmann, L., Und Lenzmann, E. Strenge Auswertung des nichtlinearen Gauß-Helmert-Modells. avn - Allgemeine Vermessungs-Nachrichten 111, 2 (2004), 68-73.

13. LöSLER, M. Zur Parameterschätzung mit unterschiedlichen Koordinatendarstellungen. zfv - Zeitschrift für Geodäsie, Geoinformation und Landmanagement (2020). doi: 10.12902/zfv-0319-2020.

14. MALISSIOVAS, G. New nonlinear adjustment approaches for applications in geodesy and related fields. Doctoral thesis, Technische Universität Berlin, Berlin, 2019. doi: 10.14279/depositonce-9194.2. 
15. Neitzel, F., Ezhov, N., Und Petrovic, S. Total least squares spline approximation. Mathematics 7, 5 (2019), 462. doi: $10.3390 /$ math7050462.

16. NITSCHKE, M., UND KNICKMEYER, E. H. Rotation parameters - a survey of techniques. Journal of Surveying Engineering 126, 3 (2000), 83-105. doi: 10.1061/(asce)0733-9453(2000)126:3(83).

17. NoCEDAL, J., UND WRIGHT, S. J. Numerical Optimization, 2. Auflage. Springer, New York, 2006. doi: 10.1007/978-0-387-40065-5.

18. WeISBACH, J. Bestimmung des Hauptstreichens und Hauptfallens von Lagerstätten. In Archiv für Mineralogie, Geognosie, Bergbau und Hüttenkunde, C. J. B. Karsten und H. v. Dechen, Eds., vol. 14. Reimer, Berlin, 1840, S. 159-174.

19. WiJeWickrema, S., Esson, C., Und PAPLIŃSki, A. Orthogonal Distance Least Squares Fitting: A Novel Approach. In Computer Vision, Imaging and Computer Graphics - Theory and Applications, A. K. Ranchordas, J. M. Pereira, H. J. Araújo, und J. M. R. S. Tavares, Eds., vol. 68. Springer, Berlin, Heidelberg, 2010, ch. Computer Vision Theory and Applications (VISAPP), pp. 255-268. doi: 10.1007/978-3-642-11840-1_19.

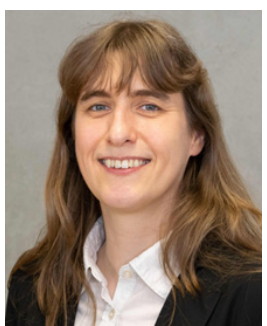

\section{Cornelia Eschelbach}

Frankfurt University of Applied Sciences, Faculty of Architecture, Civil Engineering and Geomatics, Laboratory for Industrial Metrology, Nibelungenplatz 1, D-60318 Frankfurt am Main, Germany cornelia.eschelbach@fb1.fra-uas.de
Cornelia Eschelbach studierte zwischen 1997 und 2002 Geodäsie am Karlsruher Institut für Technologie. Sie promovierte am Geodätischen Institut des Karlsruher Instituts für Technologie am Lehrstuhl für Vermessungskunde und Geodätische Sensorik von Frau Prof. Dr.-Ing Maria Hennes zum Thema: Refraktionskorrekturbestimmung durch Modellierung des Impuls- und Wärmeflusses in der Rauhigkeitsschicht. 2010 nahm sie den Ruf an die Frankfurt University of Applied Sciences für die Professur für Vermessung und angewandte Geodäsie an. Drei Jahre später gründete sie das Labor für Industrielle Messtechnik, welches in den Bereichen Large Volume Metrology und Parameterschätzung Forschungsschwerpunkte setzt.

\section{Autoreninformationen}

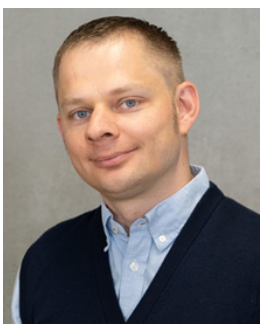

Michael Lösler

Frankfurt University of Applied Sciences, Faculty of Architecture, Civil Engineering and Geomatics, Laboratory for Industrial Metrology, Nibelungenplatz 1, D-60318 Frankfurt am Main, Germany michael.loesler@fb1.fra-uas.de

Michael Lösler schloss 2006 sein Studium zum Vermessungsingenieur an der Hochschule Neubrandenburg erfolgreich mit dem Diplom ab. Er entwickelte ein neues mathematisches Modell zur automatisierten Referenzpunktbestimmung an Radioteleskopen im Rahmen des DFG Projektes: Hochpräzise EchtzeitReferenzpunktbestimmung von VLBI-Radioteleskopen zur Verknüpfung der IVS- und IGS-Referenzrahmen am Karlsruher Institut für Technologie am Lehrstuhl für Vermessungskunde und Geodätische Sensorik von Frau Prof. Dr.-Ing Maria Hennes am Geodätischen Institut. Seit 2014 ist er im Labor für Industrielle Messtechnik der Frankfurt University of Applied Sciences beschäftigt und wissenschaftlicher Mitarbeiter im GeoMetre Projekt: Large-scale dimensional measurements for geodesy. 